\title{
OPEN Genome-wide association study of psychiatric and substance use comorbidity in Mexican individuals
}

\author{
José Jaime Martínez-Magaña ${ }^{1,2}$, Alma Delia Genis-Mendoza ${ }^{2,3}$, \\ Jorge Ameth Villatoro Velázquez ${ }^{4,5}$, Marycarmen Bustos-Gamiño ${ }^{4}$, \\ Isela Esther Juárez-Rojop ${ }^{1}$, Carlos Alfonso Tovilla-Zarate ${ }^{6}$, Emmanuel Sarmiento ${ }^{3}$, \\ Erasmo Saucedo ${ }^{7}$, Oscar Rodríguez-Mayoral ${ }^{8}$, Clara Fleiz-Bautista ${ }^{4,5}$, Beatriz Camarena ${ }^{9}$, \\ Alejandro Aguilar ${ }^{9}$, Thelma Beatriz Gonzalez-Castro ${ }^{10}$, María Elena Medina-Mora ${ }^{4,5}$ \& \\ Humberto Nicolini ${ }^{1,11 \bowtie}$
}

The combination of substance use and psychiatric disorders is one of the most common comorbidities. The objective of this study was to perform a genome-wide association study of this comorbidity (Com), substance use alone (Subs), and psychiatric symptomatology alone (Psych) in the Mexican population. The study included 3914 individuals of Mexican descent. Genotyping was carried out using the PsychArray microarray and genome-wide correlations were calculated. Genome-wide associations were analyzed using multiple logistic models, polygenic risk scores (PRSs) were evaluated using multinomial models, and vertical pleiotropy was evaluated by generalized summary-databased Mendelian randomization. Brain DNA methylation quantitative loci (brain meOTL) were also evaluated in the prefrontal cortex. Genome-wide correlation and vertical pleiotropy were found between all traits. No genome-wide association signals were found, but 64 single-nucleotide polymorphism (SNPs) reached nominal associations $(p<5.00 \mathrm{e}-05)$. The SNPs associated with each trait were independent, and the individuals with high PRSs had a higher prevalence of tobacco and alcohol use. In the multinomial models all of the PRSs (Subs-PRS, Com-PRS, and Psych-PRS) were associated with all of the traits. Brain meOTL of the Subs-associated SNPs had an effect on the genes enriched in insulin signaling pathway, and that of the Psych-associated SNPs had an effect on the Fc gamma receptor phagocytosis pathway.

Epidemiological studies have found high rates of substance use disorders in individuals with psychiatric disorders, and vice versa ${ }^{1-6}$. One characteristic of those presenting this comorbidity is an increase in the severity of the symptomatology: such patients usually present a diminished response to treatment, an exacerbation of psychotic, manic, and/or depressive symptoms, as well as an increased risk of suicide ${ }^{7-10}$, making them a highly vulnerable group $^{11}$. The etiopathological mechanisms of this comorbidity have yet to be clarified, but there are some general hypotheses ${ }^{12,13}$. One of these is the diathesis-stress model, which holds that there must be a vulnerability (such as a genetic variability) in a stressful environment (such as a socioeconomic or family situation) for a comorbidity to appear ${ }^{12}$. With the help of technologies for analyzing genetic information, genome-wide association studies have

\footnotetext{
${ }^{1}$ División Académica de Ciencias de la Salud, Universidad Juárez Autónoma de Tabasco, Villahermosa, Tabasco, Mexico. ${ }^{2}$ Laboratorio de Genómica de Enfermedades Psiquiátricas y Neurodegenerativas, Instituto Nacional de Medicina Genómica, Mexico City, Mexico. ${ }^{3}$ Hospital Psiquiátrico Infantil Juan N. Navarro, Servicios de Atención Psiquiátrica, Mexico City, Mexico. ${ }^{4}$ Unidad de Encuestas y Análisis de Datos, Instituto Nacional de Psiquiatría Ramón de la Fuente Muñiz (INPRFM), Mexico City, Mexico. ${ }^{5}$ Seminario de Estudios Sobre la Globalidad, Facultad de Medicina, Universidad Nacional Autónoma de Mexico (UNAM), Mexico City, Mexico. ${ }^{6}$ División Multidisciplinaria de Comalcalco, Universidad Juárez Autónoma de Tabasco, Comalcalco, Tabasco, Mexico. ${ }^{7}$ Centro de Neurociencias Avanzadas, Departamento de Psiquiátrica del Hospital Psiquiátrico, Universitario Dr. José Eleuterio González, Universidad Autónoma de Nuevo León, Monterrey, Nuevo León, Mexico. ${ }^{8}$ Unidad de Cuidados Paliativos, Instituto Nacional de Cancerología, Mexico City, Mexico. ${ }^{9}$ Laboratorio de Farmacogenética, Instituto Nacional de Psiquiatría Ramón de la Fuente Muñiz (INPRFM), Mexico City, Mexico. ${ }^{10}$ División Multidisciplinaria de Jalpa de Méndez, Universidad Juárez Autónoma de Tabasco, Jalpa de Méndez, Tabasco, Mexico. ${ }^{11}$ Instituto Nacional de Medicina Genómica, Periférico Sur 4809, Arenal Tepepan, Tlalpan, 14610 Mexico City, Mexico. ${ }^{\varpi}$ email: hnicolini@ inmegen.gob.mx
} 


\section{Gender}

\begin{tabular}{|l|l|l|r|}
\hline Female $(n, \%)$ & $1495(44.06)$ & $214(41.07)$ & $1709(43.66)$ \\
\hline Male $(n, \%)$ & $1898(55.94)$ & $307(58.93)$ & $2205(56.34)$ \\
\hline Age (years, mean, $s$ ) & $35.89(15.42)$ & $27.52(15.85)$ & $34.73(15.74)$ \\
\hline Comorbidity (Com, $n, \%)$ & $423(12.47)$ & $181(34.74)$ & $604(15.43)$ \\
\hline Sustance use alone $($ Subs, $n, \%)$ & $1165(34.34)$ & NA & $1165(29.76)$ \\
\hline Psychiatric symptomatology alone (Psych, $n, \%)$ & $318(9.37)$ & $340(65.26)$ & $658(16.81)$ \\
\hline No symptomatology (Cont, $n, \%)$ & $1487(43.83)$ & NA & $1487(37.99)$ \\
\hline
\end{tabular}

Table 1. Overview of the Sociodemographic and Clinical Characteristics of the Samples. ${ }^{\mathrm{a}} M x G D A R$ Mexican Genomic Database for Addiction Research. ${ }^{b} \mathrm{MeDaCros} R$ Mexican Genomic Database for Cross Disorder Research.

identified a genetic predisposition to many psychiatric disorders, demonstrating a pleiotropic effect ${ }^{14,15}$. However, these genetic studies have focused on exploring associations with a single phenotype, without investigating the question of comorbidity ${ }^{12}$. There has been little study of genetic variants related to the appearance of comorbidity.

Recent genome-wide analyses of psychiatric disorders have also included little representation of Latin American populations ${ }^{16}$. The majority of studies are of Europeans, but Latin American populations, including that of Mexico, have a high degree of genetic mixing, and could thus be the source of new associations for some phenotypes $^{17-19}$. A recent study in India, for example, found an association for schizophrenia that had not previously been reported in European populations ${ }^{20}$. Other studies have identified differences in non-Europeans from the polygenic risk scores (PRSs) derived from genetic associations based on European ancestry ${ }^{16,21}$. Because of the high degree of genetic mixing in Mexico, there is a need to find loci associated with the comorbidity. The objective of this study was thus to carry out an analysis of genome-wide association with the comorbidity of psychiatric symptomatology and substance use, substance use alone, and psychiatric symptomatology alone, in the Mexican population.

\section{Materials and methods}

Study population. This study included a total of 3914 individuals of Mexican descent, taken from two different samples: an epidemiological subsample based on population $(n=3393)$ from the Mexican Genomic Database for Addiction Research (MxGDAR), and a clinical subsample $(n=521)$ taken from the Mexican Genomic Database for Cross-Disorder Research (MeDaCrosR) (Table 1). The individuals were divided into four groups: (i) individuals with comorbidity (Com, psychiatric symptomatology and substance use), (ii) individuals with substance use alone (Subs), (iii) individuals with psychiatric symptomatology alone (Psych), and (iv) individuals without symptomatology or substance use (Cont).

The population-based epidemiological subsample was derived from the Mexican Genomic Database for Addiction Research (MxGDAR/Encodat) ${ }^{22}$, which is based on a population recruited from the 2016 National Survey of Drug, Alcohol, and Tobacco Use ${ }^{23}$. In this subsample, individuals who had experienced at least one psychiatric symptomatology in their life (psychosis, hypo/mania, depression, anxiety, or obsessions/compulsions) and presented risky use of at least one psychoactive substance (tobacco, alcohol, or drugs) were included in the Com group $(n=423)$. The psychiatric symptomatology was evaluated using the screening section from the Diagnostic Interview for Psychosis and Affective Disorders (DIP-AD) ${ }^{22,24}$. Risky substance use was determined with the questionnaire from the 2016 Survey and consisted of meeting at least one of the following criteria: (A) lifetime smoking of at least 100 cigarettes; (B) excessive alcohol consumption (at least five drinks by men or four drinks by women on a single occasion in the month prior to the survey), possible abuse or dependence on alcohol in the previous year, or having stopped drinking because of problems with its use; or (C) consumption of illegal drugs or psychoactive medications without a doctor's prescription at least six times, or consumption of at least two different illegal drugs or unprescribed medications. The Psych, Subs, and Cont groups were defined by their meeting one of the following criteria: Psych $(n=658)$ included individuals with psychiatric symptomatology but without risky substance use, Subs $(n=1165)$ included those without psychiatric symptomatology but with risky substance use, and Cont $(n=1487)$ included those with neither psychiatric symptomatology nor risky substance use.

The subsample from the Mexican Genomic Database for Cross-Disorder Research (MeDaCrosR) $(n=521)$ is a clinical sample of patients seen in different health centers in Mexico (Hospital Psiquiátrico Infantil "Juan N. Navarro," Hospital General “Dr. Gustavo A. Rovirosa Pérez,” Hospital Universitario “Dr. José Eleuterio González,” and Hospital Psiquiátrico "Fray Bernardino Alvárez"). It includes patients with diagnoses of bipolar disorder, schizophrenia, and major depressive disorder. Patients were included in the Com group $(n=181)$ if they had been diagnosed with at least one psychiatric and one substance use disorder (abuse or dependence) and they were included in the Psych group $(n=340)$ if they had been diagnosed with a psychiatric disorder but not one of substance use. The criteria used were those of the DSM-IVR, and all of the diagnoses were made by a psychiatrist. The individuals were evaluated with different psychiatric scales, including the DIGS (Diagnostic Interview for Genetic Studies) ${ }^{25}$, the MINI (Mini International Neuropsychiatric Interview) ${ }^{26}$, and the Diagnostic Interview for Psychosis and Affective Disorder (DIPAD) ${ }^{24}$. Those evaluated with the MINI were also tested for tobacco dependence with the Fagestrom test ${ }^{27}$. 
All participants provided written informed consent or assent, and the protocol complied with international norms and the Helsinki Declaration. The protocols for the MxGDAR/Encodat were reviewed and approved by the Research Ethics Committee of the Instituto Nacional de Psiquiatría (No. CEI/C/083/2015) and the Instituto Nacional de Medicina Genómica (No. 01/2017/I); the protocol for the MeDaCrosR was reviewed and approved by the Research Ethics Committee of the Instituto Nacional de Medicina Genómica (Nos. 23/2015/I and 06/2018/I).

Microarray analysis. DNA was extracted by mouth swab or from blood cells using a modified salting-out method with the Puregen commercial kit (Quiagen, USA). The quality and integrity of the DNA was analyzed with a NanoDrop spectrophotometer (Thermofisher, USA) with $2 \%$ agarose gel. Genotyping was performed with the commercial microarray Infinium PsychArray (Illumina, USA). Fluorescence intensities were read with iScan (Illumina, USA) and converted to genotypes with GenomeStudio software (Illumina, USA). Genotyping of the MxGDAR and the MeDaCrosR was carried out in the high-technology microarray unit of the Instituto Nacional de Medicina Genómica, using the same protocol.

Quality control of single nucleotide polymorphisms (SNPs). Quality control was performed with Plink software ${ }^{28}$. SNPs were excluded if they had a variant calling greater than $95 \%$, a minor allele frequency (MAF) greater than $5 \%$, a Hardy-Weinberg equilibrium chi-square test $p$-value greater than $1 \mathrm{e}-5$, and variants $\mathrm{A} / \mathrm{T}$ or $\mathrm{G} / \mathrm{C}$ (to avoid the flip strand effect). Individuals with a genotype call rate of less than $95 \%$ were excluded. To correct for cryptic relationships, all individual pairs with an identity-by-state value greater than 1.6 were marked, and the individual with the lowest genotype call rate was excluded ${ }^{29}$.

Statistical analysis. Estimation of variance and correlation at the genome-wide level. The variance explained by the SNPs was calculated using a restricted maximum-likelihood analysis (GREML) ${ }^{30,31}$ with GCTA software ${ }^{32}$. Three models of explained variance were proposed, comparing: (1) Com with Cont, (2) Psych with Cont, and (3) Subs with Cont. A bivariate GREML analysis was also performed to evaluate genome-wide correlation between Com and Subs, Com and Psych, and Subs and Psych. All of the models were adjusted for age, sex, and ten principal components of global ancestry.

Estimation of global ancestry. Estimation of global ancestry was performed with a principal components analysis using previously reported algorithms ${ }^{33}$ and the $P C-A i R$ package ${ }^{34}$. The Human Genome Diversity Project (HGDP) ${ }^{35}$ was used as a reference for population-level genotypes. Only independent SNPs were included in this estimation; for this reason a process of linkage disequilibrium pruning (LD pruning) was carried out, using the following parameters: a window size of $50 \mathrm{~kb}$, a step of 2, and a variance inflation factor of 5 .

Genome-wide genotype-phenotype associations. The genetic associations were carried out by means of multiple logistic regressions, adjusted for age, sex, and ten components of global ancestry as covariables. The logistic regressions considered (1) the Com group (MxGDAR, $n=423$, MeDaCrosR, $n=181$ ), a total of 604 cases; (2) the Subs group (MxGDAR=1165); (3) the Psych group (MxGDAR=318, MeDaCrosR=340), a total of 658 cases; and (4) the Cont group $(n=3310)$. Three statistical contrasts were performed: (a) Subs and Cont, (b) Psych and Cont; and (c) Com and Cont. A p-value of 5.00e-05 was considered nominally associated and a value of $5.00 \mathrm{e}-08$ was considered statistically significant on the genome-wide level. The logistic regressions were carried out in Plink ${ }^{28}$. After statistical contrasts, all of the variants were removed that had a MAF lower than $5.0 \%$ in cases or controls. An in silico functional annotation was also carried out of the associated SNPs using Variant Effect Predictor (VEP) ${ }^{36}$. In addition, the allelic frequencies of the associated variants were compared with the Genome Aggregation Database (GnomAD) ${ }^{37}$. A search in the GWAS atlas of the associated SNPs was performed for associations with brain-related phenotypes (psychiatric, substance use, and neurological disorders). Pathway analysis was carried out with the online ComPath tool ${ }^{38}$, using the KEGG (Kyoto Encyclopedia of Genes and Genomes) $)^{39,40}$ and Reactome databases. A clustering of paths was also performed ${ }^{41}$.

Polygenic risk scores (PRSs). Polygenic risk scores (PRSs) were calculated with SNPs associated with a significance threshold of $p<5.00 \mathrm{e}-05$ for each statistical contrast, using Plink statistical software. Three PRSs were constructed based on the following statistical contrasts: (1) Subs and Cont (Subs-PRS), (2) Psych and Cont (Psych-PRS), and (3) Com and Cont (Com-PRS). The PRS is the sum of an individual's genetic variation adjusted by the weight of every genetic variant associated with a specific $p$-value threshold ${ }^{21}$. Next, the standardized residual of the PRS was regressed on the ten principal components of global ancestry. Based on the distributions of these PRSs, the individuals were then divided into the following groups: (1) high PRS, where Com-PRS, Subs-PRS, or Psych-PRS was higher than the third quartile; (2) medium PRS, where any PRS fell between the first and third quartiles; and (3) low PRS, where any PRS was lower than the first quartile. The selected PRS was compared among the three groups (High, Medium, and Low) with an ANOVA. Next, the prevalence of psychiatric symptoms (psychosis, depression, anxiety, post-traumatic stress, obsession/compulsion, and hypo/mania) and substance use symptoms (ever used drugs, alcohol or tobacco use) was compared among the three groups with a chi-square test. These statistical comparisons were carried out with $\mathrm{R}^{42}$, and significance was established as $p<0.05$. These analyses considered only the individuals from the MxGDAR, because these had a more indepth phenotyping. Next, the accuracy of the PRSs to predict the phenotypes (Com, Subs, Psych, and Cont) was calculated with a multinomial regression using the nnet package. The sample was randomly divided into a training sample ( $70 \%$ of the individuals) and a test sample (30\% of the individuals). A multinomial regression of the phenotypes in the training sample was performed on the three PRSs constructed, and the phenotype was 
then predicted in the test sample. A contrast table of the phenotype in the test sample and those predicted by the model were compared using the caret package, and the accuracy was calculated.

Brain methylation quantitative loci (brain meQTL).. The possible functional impact of the associated SNPs on the brain was assessed by calculating brain methylation quantitative loci (brain meQTL), using a previously published database ${ }^{43-45}$ that included a total of 48 brain samples from individuals of Mexican descent. These were genotyped and DNA methylation levels were measured with microarrays. The genotyping was performed with the commercial Illumina PsychArray and the DNA methylation levels were determined using Illumina $450 \mathrm{~K}$ BeadChips (Illumina, USA). A calculation of cis- and trans- meQTL was performed with KING software $^{46}$, and $p<5.00 \mathrm{e}-8$ was considered genome-wide statistically significant.

Vertical pleiotropy. To explore whether the associated variants could perform vertical pleiotropy in the phenotypes with one exposure to an outcome, the following models were tested: (a) Subs exposure to the Com outcome, (b) Psych exposure to the Com outcome, (c) Subs exposure to the Psych outcome, (d) Psych exposure to the Subs outcome, (e) Com exposure to the Subs outcome, and (f) Com exposure to the Psych outcome. The vertical pleiotropy was tested using generalized summary-data-based Mendelian randomization (GSMR) ${ }^{47}$, with GCTA software. These analyses used SNPs in the GWAS at $p<5.0 \mathrm{e}-05$ for each genome-wide contrast (Subs and Cont, Psych and Cont, and Com and Cont).

\section{Results}

Genome-wide explained variance and correlations. The genome-wide explained variance in the Com group was $17.31 \%\left(V_{g}=0.1731\right.$, se $\left.=0.0113, p=1.16 \mathrm{e}-10\right)$, and in the Psych group it was $13.88 \%\left(V_{g}=0.1387\right.$, $s e=0.0154, p=4.44 \mathrm{e}-16)$; in the Subs group it was not statistically significant $\left(V_{g}=0.00, s e=0.0113, p=0.50\right)$. Genome-wide correlation, calculated by bivariate GREML, between Com and Psych was 0.4375 ( $s e=0.1884)$, between Com and Subs was $0.8324(s e=0.0451)$, and between Subs and Psych was $0.0486(s e=0.1542)$.

Genome-wide associations. Genome-wide association in the Subs group. The genome-wide association analysis found no genome-wide statistically significant association between Subs and Cont; however, seven SNPs reached nominal association $(p<5.00 \mathrm{e}-05)$ (Table 2a, Supplementary Table S1). Of these, three were located on intergenic regions and four on intronic regions of the LINC01622, LMO7, B3GNTL1, and C21orf91 genes. No enriched pathways were found. The only SNP reported in the GWAS atlas associated with a brain-associated phenotype was rs1304319, associated with being a morning person.

Genome-wide association in the Psych group. The comparison between Psych and Cont found a total of 40 SNPs associated at a nominal statistical level (Table 2b, Supplementary Table S2). Of these, 26 were intergenic, 13 were intronic, and one was a missense polymorphism. Associated SNPs were found on five long non-coding genes (LINC01741, LINC00578, LOC102723944, LOC105378485, and LOC105378486), eight in protein-coding regions (DPP10, PCOLCE2, SCAMP1, UBN2, NBEA, GPC6, ARHGAP23, and PTPRM); the missense polymorphism was a change of histidine for aspartic acid in the 241 position of the RGLP4 (p.His241Asp). No enriched pathways were found grouping these genes. The following SNPs were reported in the GWAS atlas as associated at a nominal genome-wide significance $(p<5.00 \mathrm{e}-05)$ to psychiatric or substance use phenotypes in other populations: rs12139234, rs10208402, rs7634577, rs13122202, rs10885147, rs4269860, rs4590798, rs3107346, rs10885243, rs1964449, rs589258, and rs608624.

The SNPs rs12139234 and rs1964449 have been associated with schizophrenia/bipolar disorder and depression, respectively, while rs10885147, rs4590798, rs589258, and rs608624 have been associated with alcohol consumption on a typical day, starting age of smoking, and frequency of memory loss due to alcohol consumption in the previous year. The following SNPs have been associated with other brain-associated phenotypes: rs 10208402 (chronotype), rs10885243 (insomnia and intelligence), rs13132202 (educational attainment), rs4269860 (right superior frontal diusivities), and rs7634577 (superior corona radiata radial diusivities).

Genome-wide association in the Com group. The comparison between Com and Cont found 17 SNPs associated at a nominally significant level (Table 2c, Supplementary Table S3). Of the 17 SNPs, seven were intergenic, nine were intronic, and one was a missense polymorphism. The nine intronic variants were located in the proteincoding genes LRRTM4, VGLL4, MGMT, LUZP2, PWP1, and FARP1, and in the LINC02067 long non-coding gene. The missense polymorphism was a change of glutamine for glutamic acid in the 215 position of the SHPK (p.Glu215Gln). No enriched pathways were found grouping these genes.

The GWAS atlas search found five SNPs (rs12747494, rs953855, rs13033902, rs971515, and rs12702917) previously associated with psychiatric or substance use phenotypes in other populations. The SNPs rs 12747494, rs971515, and rs12702917 were associated with past tobacco smoking, weekly alcohol consumption, and frequency of failure in the past year to fulfill normal expectations due to drinking, while rs953855 and rs13033902 were associated with ease of getting up in the morning and being a morning person.

PRS analysis of the different groups. The polygenic risk score analysis constructed different scores for each statistical contrast (Com and Cont, Subs and Cont, Psych and Cont), at a significance level of $p<5.00 \mathrm{e}-05$ (at this significance threshold there is no overlap of SNPs between PRSs). Subs-PRS explained $1.17 \%$ of the phenotypic variance (Pseudo- $R^{2}=0.0171, s e=0.0400, p=9.35 \mathrm{e}-09$ ), Psych-PRS explained $3.93 \%$ (Pseudo- $R^{2}=0.0393$, $s e=0.0508, p=1.61 \mathrm{e}-13$ ), and the Com-PRS explained 4.54\% (Pseudo- $R^{2}=0.0454$, $\left.s e=0.0422, p=2.42 \mathrm{e}-15\right)$. 


\begin{tabular}{|c|c|c|c|c|c|c|c|}
\hline SNP $^{\mathrm{a}}$ & Band & Position & $\mathrm{A} 1 / \mathbf{A 2}^{\mathrm{b}}$ & MAF Subs ${ }^{c}$ & MAF Cont & OR $[95 \% \mathrm{CI}] p$-value & Gene \\
\hline \multicolumn{8}{|l|}{ (a) Subs } \\
\hline rs845885 & $6 \mathrm{p} 25.3$ & 6:1019362 & $\mathrm{G} / \mathrm{T}$ & 0.3691 & 0.3186 & $\begin{array}{l}1.36[1.20-1.55] \\
1.82 \mathrm{e}-06\end{array}$ & LINC01622 (Intron) \\
\hline rs2153893 & $6 \mathrm{q} 23.3$ & 6:137295402 & $\mathrm{T} / \mathrm{C}$ & 0.2787 & 0.3264 & $\begin{array}{l}0.76[0.66-0.86] \\
4.55 \mathrm{e}-05\end{array}$ & Intergenic \\
\hline rs7048650 & $9 \mathrm{p} 22.1$ & 9:19397040 & $\mathrm{A} / \mathrm{G}$ & 0.2894 & 0.2422 & $\begin{array}{l}1.33[1.16-1.52] \\
4.26 \mathrm{e}-05\end{array}$ & \\
\hline rs9530459 & $13 \mathrm{q} 22.2$ & 13:76250576 & $\mathrm{C} / \mathrm{A}$ & 0.2174 & 0.1607 & $\begin{array}{l}1.41[1.20-1.66] \\
2.60 \mathrm{e}-05\end{array}$ & $L M O 7$ (Intron) \\
\hline rs9901757 & $17 \mathrm{q} 25.3$ & $17: 80963331$ & $\mathrm{C} / \mathrm{T}$ & 0.4264 & 0.4684 & $\begin{array}{l}0.77[0.68-0.87] \\
1.97 \mathrm{e}-05\end{array}$ & B3GNTL1 (Intron) \\
\hline rs1304319 & $18 \mathrm{p} 11.32$ & 18:1833682 & $\mathrm{T} / \mathrm{C}$ & 0.2979 & 0.2436 & $\begin{array}{l}1.33[1.16-1.52] \\
4.92 \mathrm{e}-05\end{array}$ & Intergenic \\
\hline rs2824496 & $21 \mathrm{q} 21.1$ & 21:19171547 & $\mathrm{C} / \mathrm{T}$ & 0.4569 & 0.5170 & $\begin{array}{l}0.77[0.69-0.88] \\
4.09 \mathrm{e}-05\end{array}$ & C21orf91 (Intron) \\
\hline \multicolumn{8}{|l|}{ (b) Psych } \\
\hline rs1098777 & $1 \mathrm{q} 25.2$ & 1:177508044 & $\mathrm{C} / \mathrm{T}$ & 0.2122 & 0.2637 & \begin{tabular}{|l|}
$0.71[0.60-$ \\
$0.84] 4.75 \mathrm{e}-05$ \\
\end{tabular} & Intergenic \\
\hline rs12140710 & & 1:177613863 & G/A & 0.2836 & 0.3322 & $\begin{array}{l}0.69[0.59-0.81] \\
4.35 \mathrm{e}-06\end{array}$ & \\
\hline rs4652252 & & $1: 177625201$ & G/A & 0.2353 & 0.2883 & $\begin{array}{l}0.69[0.59-0.82] \\
1.10 \mathrm{e}-05\end{array}$ & \\
\hline rs1923630 & & 1:177649849 & $\mathrm{A} / \mathrm{G}$ & 0.2583 & 0.3096 & \begin{tabular}{|l|}
$0.66[0.57-0.79]$ \\
$1.08 \mathrm{e}-06$
\end{tabular} & \\
\hline rs12139234 & & 1:177669302 & $\mathrm{A} / \mathrm{G}$ & 0.2635 & 0.3370 & $\begin{array}{l}0.71[0.61-0.83] \\
1.55 \mathrm{e}-05\end{array}$ & LINC01741 (Intron) \\
\hline rs905488 & $2 \mathrm{p} 14$ & $2: 68182025$ & $\mathrm{~T} / \mathrm{C}$ & 0.3685 & 0.4501 & $\begin{array}{l}0.72[0.63-0.84] \\
1.07 \mathrm{e}-05\end{array}$ & Intergenic \\
\hline rs10208402 & $2 \mathrm{q} 14.1$ & 2:115203917 & $\mathrm{T} / \mathrm{C}$ & 0.3247 & 0.2430 & $\begin{array}{l}1.40[1.19-1.64] \\
4.54 \mathrm{e}-05\end{array}$ & DPP10 (Intron) \\
\hline rs7634577 & $3 \mathrm{p} 25.1$ & 3:13795677 & $\mathrm{T} / \mathrm{C}$ & 0.5017 & 0.4369 & $\begin{array}{l}1.34[1.16-1.54] \\
4.67 \mathrm{e}-05\end{array}$ & Intergenic \\
\hline rs 893883 & $3 q 23$ & 3:142567327 & $\mathrm{A} / \mathrm{G}$ & 0.4414 & 0.3592 & $\begin{array}{l}1.37[1.18-1.59] \\
3.97 \mathrm{e}-05\end{array}$ & PCOLCE2 (Intron) \\
\hline rs6440116 & & 3:142576455 & $\mathrm{T} / \mathrm{C}$ & 0.4396 & 0.3592 & $\begin{array}{l}1.38[1.18-1.60] \\
3.14 \mathrm{e}-05\end{array}$ & \\
\hline rs13074870 & $3 q 26.32$ & 3:177363666 & $\mathrm{C} / \mathrm{T}$ & 0.4077 & 0.3303 & $\begin{array}{l}1.36[1.18-1.57] \\
2.88 \mathrm{e}-05\end{array}$ & LINC00578 (Intron) \\
\hline rs6789946 & & $3: 177374031$ & $\mathrm{C} / \mathrm{T}$ & 0.3459 & 0.2803 & \begin{tabular}{|l|}
$1.36[1.17-1.60]$ \\
$3.97 \mathrm{e}-05$ \\
\end{tabular} & \\
\hline rs2049156 & $4 \mathrm{p} 15.1$ & 4:29018173 & $\mathrm{A} / \mathrm{G}$ & 0.2571 & 0.1976 & $\begin{array}{l}1.43[1.21-1.69] \\
3.30 \mathrm{e}-05\end{array}$ & Intergenic \\
\hline rs11097166 & $4 \mathrm{q} 22.1$ & $4: 88611344$ & G/A & 0.4866 & 0.4074 & $\begin{array}{l}1.41[1.22-1.62] \\
2.66 \mathrm{e}-06\end{array}$ & \\
\hline rs13132202 & $4 \mathrm{q} 34.3$ & 4:179125887 & $\mathrm{C} / \mathrm{T}$ & 0.2052 & 0.2601 & $\begin{array}{l}0.69[0.58-0.82] \\
1.68 \mathrm{e}-05\end{array}$ & \\
\hline rs7701154 & $5 q 14.1$ & 5:77716621 & $\mathrm{A} / \mathrm{G}$ & 0.5128 & 0.4360 & $\begin{array}{l}1.37[1.18-1.60] \\
3.88 \mathrm{e}-05\end{array}$ & $S C A M P 1$ (Intron) \\
\hline rs3860112 & $5 q 31.1$ & 5:134893439 & $\mathrm{C} / \mathrm{T}$ & 0.4464 & 0.5088 & $\begin{array}{l}0.73[0.63-0.84] \\
1.47 \mathrm{e}-05\end{array}$ & Intergenic \\
\hline rs2317965 & $6 \mathrm{p} 25.3$ & 6:1545513 & G/A & 0.5461 & 0.4800 & $\begin{array}{l}1.35[1.17-1.55] \\
3.63 \mathrm{e}-05\end{array}$ & $\begin{array}{l}\text { LOC102723944 } \\
\text { (Intron) }\end{array}$ \\
\hline rs7796255 & $7 \mathrm{q} 34$ & 7:138963364 & $\mathrm{A} / \mathrm{C}$ & 0.2705 & 0.1940 & $\begin{array}{l}1.46[1.23-1.73] \\
1.19 \mathrm{e}-05 \\
\end{array}$ & UBN2 (Intron) \\
\hline rs10885147 & $10 \mathrm{q} 25.2-10 \mathrm{q} 26.13$ & 10:113144540 & $\mathrm{T} / \mathrm{G}$ & 0.2906 & 0.3288 & \begin{tabular}{|l|}
$0.71[0.61-0.84]$ \\
$3.69 \mathrm{e}-05$
\end{tabular} & Intergenic \\
\hline rs4269860 & & 10:113180191 & $\mathrm{C} / \mathrm{T}$ & 0.3250 & 0.3627 & $\begin{array}{l}0.71[0.61-0.84] \\
2.65 \mathrm{e}-05\end{array}$ & \\
\hline rs4590798 & & 10:113194854 & G/A & 0.2831 & 0.3217 & $\begin{array}{l}0.71[0.61-0.84] \\
4.19 \mathrm{e}-05\end{array}$ & $\begin{array}{l}\text { LOC105378485 } \\
\text { (Intron) }\end{array}$ \\
\hline rs11527950 & & 10:113248666 & $\mathrm{C} / \mathrm{T}$ & 0.2680 & 0.3100 & $\begin{array}{l}0.70[0.59-0.82] \\
1.81 \mathrm{e}-05\end{array}$ & Intergenic \\
\hline rs3107346 & & 10:113261163 & $\mathrm{A} / \mathrm{G}$ & 0.3406 & 0.3891 & $\begin{array}{l}0.71[0.61-0.83] \\
1.87 \mathrm{e}-05\end{array}$ & \\
\hline rs11195620 & & 10:113287674 & $\mathrm{T} / \mathrm{C}$ & 0.3792 & 0.4284 & \begin{tabular}{|l|}
$0.70[0.60-0.82]$ \\
$6.39 \mathrm{e}-06$
\end{tabular} & \\
\hline rs2138554 & & 10:113310787 & $\mathrm{T} / \mathrm{G}$ & 0.2953 & 0.3452 & \begin{tabular}{|l|}
$0.69[0.59-0.81]$ \\
$4.64 \mathrm{e}-06$
\end{tabular} & \\
\hline
\end{tabular}




\begin{tabular}{|c|c|c|c|c|c|c|c|}
\hline SNP $^{\mathrm{a}}$ & Band & Position & $\mathrm{A} 1 / \mathrm{A} 2^{\mathrm{b}}$ & MAF Subs ${ }^{c}$ & MAF Cont & OR $[95 \% \mathrm{CI}] p$-value & Gene \\
\hline rs 10885243 & & 10:113445582 & $\mathrm{A} / \mathrm{G}$ & 0.3107 & 0.3481 & $\begin{array}{l}0.72[0.62-0.84] \\
4.84 \mathrm{e}-05\end{array}$ & $\begin{array}{l}\text { LOC105378486 } \\
\text { (Intron) }\end{array}$ \\
\hline rs1797 & & 10:119705438 & $\mathrm{T} / \mathrm{C}$ & 0.4264 & 0.3513 & $\begin{array}{l}1.36[1.18-1.58] \\
3.95 \mathrm{e}-05\end{array}$ & Intergenic \\
\hline rs10901874 & & 10:126895917 & $\mathrm{T} / \mathrm{C}$ & 0.2647 & 0.2017 & $\begin{array}{l}1.42[1.20-1.67] \\
3.74 \mathrm{e}-05\end{array}$ & \\
\hline rs11608012 & $11 \mathrm{p} 15.1$ & $11: 21617059$ & $\mathrm{~A} / \mathrm{G}$ & 0.3874 & 0.3088 & $\begin{array}{l}1.42[1.22-1.65] \\
3.74 \mathrm{e}-05\end{array}$ & \\
\hline rs1783235 & $11 \mathrm{q} 23.3$ & 11:116050271 & G/A & 0.2998 & 0.3612 & $\begin{array}{l}0.72[0.62-0.84] \\
3.25 \mathrm{e}-05\end{array}$ & \\
\hline rs1824603 & $13 \mathrm{q} 13.3$ & 13:35955344 & G/A & 0.2454 & 0.1834 & $\begin{array}{l}1.45[1.23-1.72] \\
1.37 \mathrm{e}-05\end{array}$ & NBEA (Intron) \\
\hline rs1964449 & $13 \mathrm{q} 14.3$ & 13:54034731 & $\mathrm{A} / \mathrm{G}$ & 0.5226 & 0.4519 & $\begin{array}{l}1.34[1.17-1.55] \\
4.69 \mathrm{e}-05\end{array}$ & Intergenic \\
\hline rs589258 & $13 \mathrm{q} 31.3$ & 13:94807356 & $\mathrm{T} / \mathrm{C}$ & 0.3585 & 0.2929 & $\begin{array}{l}1.37[1.18-1.59] \\
4.08 \mathrm{e}-05\end{array}$ & GPC6 (Intron) \\
\hline rs608624 & $17 \mathrm{q} 11.2$ & $17: 16404247$ & $\mathrm{~T} / \mathrm{C}$ & 0.2956 & 0.2184 & $\begin{array}{l}1.44[1.22-1.70] \\
1.77 \mathrm{e}-05\end{array}$ & Intergenic \\
\hline rs8067381 & $17 q 12$ & $17: 36578907$ & $\mathrm{~T} / \mathrm{C}$ & 0.1315 & 0.0797 & $\begin{array}{l}1.64[1.30-2.07] \\
3.44 \mathrm{e}-05\end{array}$ & ARHGAP23 (Intron) \\
\hline rs 2027670 & $18 \mathrm{p} 11.23$ & $18: 7732141$ & $\mathrm{G} / \mathrm{T}$ & 0.3865 & 0.4691 & $\begin{array}{l}0.70[0.61-0.82] \\
3.52 \mathrm{e}-06\end{array}$ & PTPRM (Intron) \\
\hline rs658864 & & $18: 7734917$ & G/A & 0.4231 & 0.3454 & $\begin{array}{l}1.41[1.22-1.64] \\
6.16 \mathrm{e}-06\end{array}$ & \\
\hline rs762416 & $21 \mathrm{q} 22.3$ & $21: 45267134$ & $\mathrm{~T} / \mathrm{C}$ & 0.3291 & 0.4075 & \begin{tabular}{|l|}
$0.71[0.62-0.83]$ \\
$6.11 \mathrm{e}-06$ \\
\end{tabular} & Intergenic \\
\hline rs2070446 & $22 \mathrm{q} 11.23$ & $22: 24035970$ & $\mathrm{C} / \mathrm{T}$ & 0.2605 & 0.2044 & $\begin{array}{l}1.41[1.20-1.67] \\
4.88 \mathrm{e}-05\end{array}$ & RGLP4 (p.His241Asp) \\
\hline \multicolumn{8}{|l|}{ (c) Com } \\
\hline rs12747494 & $1 \mathrm{p} 22.2$ & $1: 91944137$ & $\mathrm{C} / \mathrm{T}$ & 0.2354 & 0.1910 & $\begin{array}{l}1.56[1.30-1.86] \\
1.40 \mathrm{e}-06\end{array}$ & Intergenic \\
\hline rs953855 & $2 \mathrm{p} 12$ & $2: 77123866$ & $\mathrm{G} / \mathrm{T}$ & 0.4630 & 0.4912 & $\begin{array}{l}0.71[0.62-0.83] \\
1.29 \mathrm{e}-05\end{array}$ & LRRTM4 (Intron) \\
\hline rs 13033902 & & $2: 77264354$ & $\mathrm{C} / \mathrm{T}$ & 0.1952 & 0.2453 & $\begin{array}{l}0.68[0.57-0.82] \\
3.46 \mathrm{e}-05\end{array}$ & \\
\hline rs4848094 & & $2: 121400276$ & $\mathrm{C} / \mathrm{T}$ & 0.3853 & 0.3193 & $\begin{array}{l}1.40[1.20-1.63] \\
2.53 \mathrm{e}-05\end{array}$ & Intergenic \\
\hline rs1039201 & $3 p 25.3$ & $3: 11731029$ & $\mathrm{~T} / \mathrm{C}$ & 0.4212 & 0.4705 & \begin{tabular}{|l|}
$0.73[0.63-0.84]$ \\
$3.50 \mathrm{e}-05$
\end{tabular} & VGLL4 (Intron) \\
\hline rs971515 & $3 q 26.1$ & 3:161153006 & $\mathrm{C} / \mathrm{T}$ & 0.4863 & 0.4016 & $\begin{array}{l}1.37[1.18-1.58] \\
2.81 \mathrm{e}-05\end{array}$ & LINC02067 (Intron) \\
\hline rs10076602 & $5 q 33.2$ & 5:153902050 & $\mathrm{T} / \mathrm{G}$ & 0.1096 & 0.1306 & \begin{tabular}{|l|}
$0.61[0.48-0.77]$ \\
$4.24 \mathrm{e}-05$
\end{tabular} & Intergenic \\
\hline rs12702917 & $7 \mathrm{p} 21.3$ & 7:9550478 & $\mathrm{A} / \mathrm{G}$ & 0.4513 & 0.3606 & $\begin{array}{l}1.38[1.19-1.61] \\
3.67 \mathrm{e}-05\end{array}$ & \\
\hline rs887060 & $7 \mathrm{p} 14.1$ & $7: 43057398$ & $\mathrm{C} / \mathrm{T}$ & 0.3010 & 0.3614 & $\begin{array}{l}0.72[0.62-0.83] \\
3.14 \mathrm{e}-05\end{array}$ & \\
\hline rs4470979 & $7 \mathrm{p} 11.2$ & $7: 57320738$ & $\mathrm{~A} / \mathrm{G}$ & 0.1852 & 0.2218 & $\begin{array}{l}0.66[0.55-0.80] \\
2.19 \mathrm{e}-05\end{array}$ & \\
\hline rs7078706 & $10 \mathrm{q} 26.3$ & 10:131525017 & $\mathrm{T} / \mathrm{G}$ & 0.2847 & 0.2103 & $\begin{array}{l}1.51[1.27-1.78] \\
1.94 \mathrm{e}-06\end{array}$ & $M G M T$ (Intron) \\
\hline rs7118149 & $11 \mathrm{p} 14.3$ & 11:24700545 & $\mathrm{A} / \mathrm{G}$ & 0.4675 & 0.3776 & $\begin{array}{l}1.41[1.21-1.65] \\
8.86 \mathrm{e}-06\end{array}$ & LUZP2 (Intron) \\
\hline rs10778569 & $12 \mathrm{q} 23.3$ & 12:108087904 & G/A & 0.2659 & 0.3315 & $\begin{array}{l}0.68[0.57-0.81] \\
4.92 \mathrm{e}-06\end{array}$ & $P W P 1$ (Intron) \\
\hline rs6491403 & $13 q 32.2$ & 13:98866841 & $\mathrm{A} / \mathrm{G}$ & 0.4700 & 0.4166 & $\begin{array}{l}1.37[1.18-1.58] \\
3.62 \mathrm{e}-05\end{array}$ & FARP1 (Intron) \\
\hline rs 4238213 & & 13:98869342 & $\mathrm{T} / \mathrm{G}$ & 0.5094 & 0.4436 & $\begin{array}{l}1.42[1.22-1.65] \\
3.65 \mathrm{e}-06\end{array}$ & \\
\hline rs11071657 & $15 \mathrm{q} 22.2$ & $15: 62433962$ & $\mathrm{~A} / \mathrm{G}$ & 0.5146 & 0.4240 & $\begin{array}{l}1.36[1.18-1.58] \\
4.47 \mathrm{e}-05\end{array}$ & Intergenic \\
\hline rs150857 & & $20: 62173817$ & $\mathrm{~T} / \mathrm{C}$ & 0.1355 & 0.1851 & $\begin{array}{l}0.31[0.18-0.52] \\
1.41 \mathrm{e}-05\end{array}$ & SHPK (p.Glu215Gln) \\
\hline
\end{tabular}

Table 2. Genetic loci associated with Subs, Psych, and Com. a'Single-nucleotide variant, dbSNP code. ${ }^{\mathrm{b}} \mathrm{A} 1=$ effect allele/A2 $=$ no effect allele. ${ }^{\mathrm{c}}$ Minor allele frequency of A1 allele. ${ }^{\mathrm{d} E f f e c t}$, in silico predicted effect of the variant. 
\begin{tabular}{|l|l|l|l} 
High PRS $(n=1950)$ & Medium PRS $(n=399)$ & Low PRS $(n=1044)$ & Statistic $(p)$ \\
\hline
\end{tabular}

Polygenic risk scores

\begin{tabular}{|l|l|l|l|l|}
\hline Comorbidity (Com-PRS) & $0.30(1.07)$ & $-0.02(0.37)$ & $-0.60(0.73)$ & $15.63(4.26 \mathrm{e}-10)$ \\
\hline Substance use (Subs-PRS) & $0.31(1.08)$ & $-0.01(0.35)$ & $-0.59(0.69)$ & $11.58(1.51 \mathrm{e}-07)$ \\
\hline Psychiatric (Psych-PRS) & $0.30(1.05)$ & $-0.01(0.35)$ & $-0.62(0.72)$ & $13.34(1.19 \mathrm{e}-08)$ \\
\hline Psch
\end{tabular}

\begin{tabular}{|l|l|l|l|}
\hline Psychiatric symptoms & \multicolumn{2}{l}{} \\
\hline Hypo(manta) & & \\
\hline
\end{tabular}

\begin{tabular}{|l|c|c|c|l|}
\hline Hypo(mania) & $194(9.95)$ & $46(11.53)$ & $90(8.62)$ & $3.50(0.1740)$ \\
\hline Post-traumatic stress & $144(7.38)$ & $28(7.02)$ & $56(5.36)$ & $4.38(0.1118)$ \\
\hline Depression & $166(8.51)$ & $31(7.77)$ & $64(6.13)$ & $5.23(0.0733)$ \\
\hline Anxiety & $131(6.72)$ & $16(4.01)$ & $48(4.60)$ & $7.53(0.0232)$ \\
\hline Obsession/compulsion & $71(3.64)$ & $10(2.51)$ & $27(2.60)$ & $2.85(0.2396)$ \\
\hline Psychosis & $37(1.90)$ & $4(1.00)$ & $12(1.15)$ & $3.19(0.2028)$ \\
\hline
\end{tabular}

Substance use

\begin{tabular}{|l|c|c|c|l|}
\hline Alcohol $^{\mathrm{a}}$ & $765(39.23)$ & $151(37.84)$ & $332(31.80)$ & $15.94(3.45 \mathrm{e}-04)$ \\
\hline Tobacco $^{\mathrm{b}}$ & $489(25.08)$ & $92(23.06)$ & $198(18.97)$ & $13.73(1.04 \mathrm{e}-03)$ \\
\hline Cannabis & $331(16.97)$ & $57(14.29)$ & $156(14.94)$ & $2.42(0.2978)$ \\
\hline Cocaine & $138(7.08)$ & $24(6.02)$ & $74(7.09)$ & $0.40(0.8174)$ \\
\hline Tranquilizers & $38(1.95)$ & $10(2.51)$ & $15(1.44)$ & $2.18(0.3366)$ \\
\hline Crack & $35(1.79)$ & $8(2.00)$ & $20(1.92)$ & $0.17(0.9186)$ \\
\hline Inhalants & $36(1.85)$ & $9(2.26)$ & $14(1.34)$ & $1.83(0.4003)$ \\
\hline Hallucinogens & $23(1.18)$ & $4(1.00)$ & $12(1.15)$ & $0.06(0.9722)$ \\
\hline Amphetamines & $14(0.72)$ & $4(1.00)$ & $8(0.77)$ & $0.43(0.8062)$ \\
\hline Heroin & $8(0.41)$ & $3(0.75)$ & $3(0.29)$ & $1.63(0.4434)$ \\
\hline Opioids & $4(0.21)$ & $0(0.00)$ & $5(0.48)$ & $3.12(0.2100)$ \\
\hline Sedatives & $4(0.21)$ & $1(0.25)$ & $1(0.09)$ & $0.61(0.7364)$ \\
\hline
\end{tabular}

Table 3. Psychiatric symptoms and substance use in the MxGDAR subsample with different PRSs. ${ }^{\text {aExcessive }}$ alcohol consumption, blifetime smoking of at least 100 cigarettes.

In the multinomial model, there was a statistically significant association of the three PRSs with every group (Supplementary Table S1). The three PRSs had an accuracy of $40.69 \%\left(\mathrm{CI}_{95 \%} 37.81-43.62 \%\right)$ for the prediction of Com, Subs, and Psych. Next, the individuals were grouped based on PRS, and the differences in psychiatric symptoms and substance use patterns in individuals with different PRSs were evaluated (Table 3); these analyses included only individuals from the MxGDAR.

There were statistically significant differences between individuals with different PRSs in lifetime prevalence of anxiety, lifetime smoking of at least 100 cigarettes, and problematic use of alcohol (excessive alcohol consumption, possible abuse or dependence on alcohol in the previous year, or having stopped drinking because of problems with its use).

Brain meQTL analysis of associated variants. The brain meQTLs associated with Subs, rs4787483, rs1304319, rs2824496, and rs9901757, had an effect on 269 CpG sites, 48 of which were annotated to the gene promoter (Table 4a). The greatest association was for rs2824496 affecting cg21278102, annotated to the promoter of HSBP1 (Table 4a). The genes affected by the brain meQTL associated with Subs were enriched for the insulin signaling pathway (hsa04910, adjusted $p=0.0225$ ) and the mTOR signaling pathway (hsa04150, adjusted $\mathrm{p}=0.0269)$. Of the 40 SNPs associated with Psych, 19 (rs10208402, rs10885147, rs10885243, rs1098777, rs11527950, rs12140710, rs13074870, rs1923630, rs2049156, rs2317965, rs4269860, rs4590798, rs4652252, rs589258, rs608624, rs6789946, rs762416, rs7701154, and rs905488) had a cis or trans effect on 643 CpGs sites, of which 61 were annotated to the gene promoter, with the greatest effect for the rs 13074870 associated with cg26017930, annotated to SKI (Table 4b). The brain meQTLs associated with Psych were enriched for 50 pathways, including Axon guidance (hsa04360, adjusted $p<1.00 \mathrm{e}-4)$ and Fc gamma R-mediated phagocytosis (hsa04666, adjusted $p=1.00 \mathrm{e}-04$ ). Of the 17 SNPs associated with the Com, seven (rs12747494, rs1039201, rs4470979, rs7078706, rs7118149, rs953855, and rs971515) had an effect on 91 CpGs sites, of which 25 were associated with the gene promoter. In these associations the greatest effects were seen by the rs12747494 affecting cg25100604 and cg275519869, CpGs sites annotated to the promoters of PCBP1 and FAM133B, respectively (Table 4c). There were no enriched pathways for the genes where the brain meQTL associated with Com had an effect.

Vertical pleiotropy. Vertical pleiotropy between phenotypes was assessed at $p<5.00 \mathrm{e}-05$ for the associated SNPs. Vertical pleiotropy was found in both exposures: Psych to Com $(b x y=0.3004, s e=0.0675, p=8.61 \mathrm{e}-06$, $n S N P s=12)$ and Subs to Com $(b x y=0.4079, s e=0.1109, p=2.35 \mathrm{e}-04, n S N P s=7)$. Reverse vertical pleiotropy was also found in Com to Psych (bxy=0.3065, se=0.0803, $p=1.37 \mathrm{e}-04, n S N P s=10)$ and Com to Subs $(b x y=0.3724$, $s e=0.0747, p=6.11 \mathrm{e}-07, n S N P s=10)$. There was vertical pleiotropy in Subs to Psych $(b x y=0.3224, s e=0.1056$, 


\begin{tabular}{|c|c|c|c|c|c|c|c|}
\hline SNP & SNP position & CpG & CpG position & CpG gene & Beta & se & $p$-value \\
\hline \multicolumn{8}{|l|}{ (a) Subs } \\
\hline rs 4787483 & $16: 29885447$ & $\operatorname{cg} 17945962$ & $2: 67793216$ & - & 1.8154 & 0.3932 & $3.80 \mathrm{e}-06$ \\
\hline rs2824496 & 21:19171547 & cg21278102 & 19:60482693 & $H S B P 1$ (promoter) & -1.2032 & 0.2537 & $2.10 \mathrm{e}-06$ \\
\hline \multicolumn{8}{|l|}{ (b) Psych } \\
\hline rs10208402 & 2:115203917 & cg09191731 & $16: 22825045$ & HS3ST2 & 1.3293 & 0.2874 & $3.73 e-06$ \\
\hline \multirow[t]{23}{*}{ rs13074870 } & 3:177363666 & cg26017930 & $1: 2232165$ & SKI (Promoter) & 1.2075 & 0.2555 & $2.28 \mathrm{e}-06$ \\
\hline & & cg13906646 & 6:166892920 & RPS6KA2 & -1.2005 & 0.2555 & $2.61 \mathrm{e}-06$ \\
\hline & & cg26559829 & $16: 85206154$ & & -1.1834 & 0.2555 & $3.61 \mathrm{e}-06$ \\
\hline & & $\operatorname{cg} 06852461$ & 1:207975183 & C1orf132 & -1.1825 & 0.2555 & $3.67 \mathrm{e}-06$ \\
\hline & & $\operatorname{cg} 04144365$ & $2: 188483529$ & & -1.2275 & 0.2555 & $1.55 \mathrm{e}-06$ \\
\hline & & $\operatorname{cg} 06709297$ & $2: 10302453$ & C2orf 48 & -1.2226 & 0.2555 & $1.70 \mathrm{e}-06$ \\
\hline & & cg07046426 & $2: 237623902$ & & 1.1832 & 0.2555 & $3.62 \mathrm{e}-06$ \\
\hline & & cg10464312 & $2: 66672687$ & MEIS1 & 1.1709 & 0.2555 & $4.57 \mathrm{e}-06$ \\
\hline & & cg06055229 & 5:124077965 & ZNF608 & -1.1714 & 0.2555 & $4.53 \mathrm{e}-06$ \\
\hline & & cg06445944 & $5: 67586927$ & PIK3R1 & -1.1734 & 0.2555 & $4.36 \mathrm{e}-06$ \\
\hline & & cg14196170 & 6:32063594 & TNXB & -1.2095 & 0.2555 & $2.19 \mathrm{e}-06$ \\
\hline & & cg26577169 & 6:166401611 & LINC00602 & -1.1663 & 0.2555 & $4.98 \mathrm{e}-06$ \\
\hline & & cg16107105 & $7: 150646703$ & KCNH2 & 1.1754 & 0.2555 & $4.20 \mathrm{e}-06$ \\
\hline & & cg09759289 & $8: 28223456$ & FBXO16 & -1.1725 & 0.2555 & $4.43 \mathrm{e}-06$ \\
\hline & & cg26280695 & 10:106072439 & ITPRIP & 1.1991 & 0.2555 & $2.68 \mathrm{e}-06$ \\
\hline & & $\operatorname{cg} 03447547$ & 14:94577038 & IFI27 & 1.2104 & 0.2555 & $2.16 \mathrm{e}-06$ \\
\hline & & $\operatorname{cg} 19733938$ & 14:102510359 & DYNC1H1 & -1.1678 & 0.2555 & $4.84 \mathrm{e}-06$ \\
\hline & & cg23909173 & 14:94451388 & & -1.1927 & 0.2555 & $3.03 \mathrm{e}-06$ \\
\hline & & $\operatorname{cg} 05333442$ & $16: 58533742$ & NDRG4 & -1.1678 & 0.2555 & $4.84 \mathrm{e}-06$ \\
\hline & & $\operatorname{cg} 00080125$ & $17: 59490612$ & C17orf82 & 1.1733 & 0.2555 & $4.37 \mathrm{e}-06$ \\
\hline & & $\operatorname{cg} 00591333$ & 17:79109909 & AATK & -1.1854 & 0.2555 & $3.48 \mathrm{e}-06$ \\
\hline & & cg19919217 & $17: 38248104$ & THRA & -1.2131 & 0.2555 & $2.05 \mathrm{e}-06$ \\
\hline & & cg14771451 & $22: 18508297$ & MICAL3 & -1.1858 & 0.2555 & $3.45 \mathrm{e}-06$ \\
\hline rs7701154 & 5:77716621 & cg18281939 & $5: 77783894$ & LHFPL2 & -1.1750 & 0.2495 & $2.49 \mathrm{e}-06$ \\
\hline \multicolumn{8}{|l|}{ (c) Com } \\
\hline \multirow[t]{3}{*}{ rs12747494 } & $1: 91944137$ & cg25100604 & 2:70314405 & PCBP1 (Promoter) & -1.3127 & 0.2844 & $3.91 \mathrm{e}-06$ \\
\hline & & cg08057136 & 2:207506976 & - & -1.3785 & 0.2844 & $1.25 \mathrm{e}-06$ \\
\hline & & cg27519869 & 7:92219804 & FAM133B (Promoter) & -1.3326 & 0.2844 & $2.78 \mathrm{e}-06$ \\
\hline rs953855 & $2: 77123866$ & $\operatorname{cg} 13294780$ & 4:140005755 & $E L F 2$ & -1.1535 & 0.2494 & $3.74 \mathrm{e}-06$ \\
\hline rs1039201 & $3: 11731029$ & $\operatorname{cg} 10087374$ & $8: 21960455$ & FAM160B2 & 1.1584 & 0.2500 & $3.61 \mathrm{e}-06$ \\
\hline
\end{tabular}

Table 4. Brain methylation quantitative loci (meQTLs) of the associated SNPs.

$p=2.26 \mathrm{e}-03, n S N P s=7)$, and reverse vertical pleiotropy in Psych to Subs $(b x y=0.2953, s e=0.0629, p=2.69 \mathrm{e}-06$, $n S N P s=12)$. Of the 64 SNPs associated with any phenotype at nominal significance, $29(45.32 \%)$ showed vertical pleiotropy of any type.

\section{Discussion}

The comorbidity between psychiatric symptomatology and substance use leads to a significant impairment in affected individuals, but the evaluation of the phenotype has been little explored in genome-wide studies. Ours is one of the first genome-wide association studies to explore variants associated with this comorbidity, along with substance use and psychiatric symptoms alone, in the Mexican population.

Our study found that the evaluation of different phenotypes (Subs, Psych, and Com) could identify different patterns of associated variants, but that these associated variants are highly correlated and could also have pleiotropy, the effect of a single variant on different phenotypes or traits ${ }^{48}$. Genome-wide association studies have found a high degree of correlation between psychiatric disorders ${ }^{49}$, possibly because many of the associated genes could have pleiotropic effects between different psychiatric phenotypes. In this study we found vertical pleiotropy and genome-wide correlation of all phenotypes, suggesting that the associated variants could have an effect in Subs, Psych, and Com. These have been reported recently in a genome-wide study of lifelong cannabis use, which found a genetic correlation of the phenotype with different substance use disorders as well as with other mental disorders, meaning that many of the associated genes could show a pleiotropic effect in these phenotypes ${ }^{15}$. Other studies have suggested that the categorization of individuals into discrete diagnoses may neglect the consideration of these individuals in terms of a broader phenotype, which may be occurring in comorbidity studies ${ }^{50-54}$. The use of genetic analysis could help us to better categorize individuals with comorbidity, as in our finding that 
individuals with higher polygenic risk scores had a higher prevalence of having smoked more than 100 cigarettes in their life and a higher prevalence of risky alcohol use. Interestingly, the variants found in the PRS analysis to be associated with Com and Psych have been associated in the GWAS atlas with alcohol and tobacco phenotypes. This may be the result of the larger sample size in our study of individuals using alcohol or tobacco. We thus believe that genome-wide association studies of comorbidity might include a greater diversity of substance use disorders in order to explore this phenotype-dependent difference in GWAS signals. If so, PRS results could be used in a clinical setting to screen for individuals with a greater risk of developing the comorbidity.

In order to assess the possible effects of the associated SNPs on the brain, we performed brain meQTL analysis of a previously published database of individuals of Mexican ancestry ${ }^{43-45}$. Some polymorphisms showed greater evidence of association with brain phenotypes, including rs10208402, rs12747494, and rs953855. The first, the intron SNP in the gene DPP10, for which we found an association with psychiatric disorders, has been associated with chronotype ${ }^{55-57}$. This variant exerted a trans effect on a CpG site on gene HS3ST2, also associated with chronotype ${ }^{55}$. We also found an association of rs953855, the SNP in the intron of LRRTM4, with the Com; it too has been associated with chronotype. A recent gene-level analysis found an association of the LRRTM4 loci with lifetime cannabis use ${ }^{15}$. These results suggest that the comorbidity could be associated with chronotype. The rs953855 in this study was a brain meQTL, associated in trans to a CpG site in the ELF2 gene, which has been suggested as a sensor for the elevation of extrasynaptic glutamate, modifying the growth of functional dendritic spines ${ }^{58}$. Glutamatergic signaling is a regulator in the reward regions of the brain that maintain the habits of psychoactive substance use ${ }^{59}$. The use of such substances alters this signaling; the mechanisms depend on the substance used, but the great majority promote an increase in glutamate at the synaptic level, which leads to an increase in the activation of neuronal receptors and a glutamate-dependent excitotoxicity, a mechanism dependent on $\mathrm{Ca}^{2+60-64}$. The increase in excitotoxicity could generate changes in neuroplasticity, leading to an increase in drug-seeking behaviors and in the memories associated with drugs ${ }^{65}$. The intergenic SNP, rs127474, for which we found an association with the comorbidity, is also associated with ever/never used tobacco ${ }^{57}$. This SNP had a trans effect on two CpG sites located in the promoters on PCBP1 and FAM133B. PCBP1 is part of the DISC1 interactome ${ }^{66}$, which is essential in the development of brain cells, and alterations in this area could lead to neurodegenerative disorders ${ }^{66-68}$. Peripheral levels of DISC1 have been proposed as a marker of nicotine exposure $^{69}$, supporting the possibility of an association between rs 127474 and tobacco-related behavior.

On the pathway/functional level, we found that brain meQTLs associated with Subs and Psych have a greater effect on brain pathways than those associated with Com. Those associated with Subs modified the insulin signaling pathway, while those associated with Psych modified the Fc gamma receptor mediated phagocytosis. Insulin signaling in the periphery plays a crucial role in the homeostasis of plasma glucose levels, but the effect of insulin on the central nervous system is less recognized. Insulin in the CNS is involved in cell survival, neurogenesis, receptor trafficking, and neurotransmitter release/reuptake $\mathrm{e}^{70-73}$. The insulin pathway has been associated with substance addiction in animal and human studies, and in integrative bioinformatics analysis of different omic data $^{74}$. The mechanism underlying the effect of insulin in substance use is not fully elucidated, but evidence points to a dysregulation of dopamine in brain reward circuits ${ }^{75-79}$. The Fc gamma receptor mediated phagocytosis pathway, for which we found brain meQTLs associated with psychiatric symptoms, was recently associated with schizophrenia and bipolar disorder through analysis of transcriptomes and co-localization of GWAS signals ${ }^{80}$. Zhao et al. have suggested that the alteration of the Fc gamma receptor pathway could affect lysosomal function, and found that individuals with lysosomal dysfunction had greater manifestations of psychiatric symptoms ${ }^{81}$, suggesting its possible importance in the manifestation of this symptomatology.

The genome-wide estimated variance in the Com group was greater than in those who presented only one disorder, suggesting that the risk from genetic variability could be greater in this group. Interestingly, the explained variance in the Subs group was close to zero. This lesser explained variance could mean that the effects of the common variants analyzed in the microarray are not sufficient to capture the genetic effect in this group, possibly because of an underestimation, possibly because of the effects of uncommon variants not explored in this study. An examination of these phenotypes with other sources of genetic variation is thus needed, and of the way in which this genetic risk could interact with environmental factors such as exposure to trauma and social tolerance of substance use in producing the comorbidity.

Although this study identified associations that could be the basis for future functional studies of the relationship between genetic variability and comorbidity, some limitations should be noted. The main one is the use of two samples, one a population sample and the other clinical, where the criteria for defining the phenotype could be a source of heterogeneity. However, the inclusion of both populations increases the sample size and facilitates the identification of associations. Even with these limitations, we believe that this study, with the sample size it offers for investigation of the genome-wide association, provides important information for the understanding of the comorbidity between psychiatric symptomatology and substance use in the Mexican population.

This study found genetic associations of SNPs that modulate brain DNA methylation levels in genes involved in the insulin signaling pathway and $\mathrm{Fc}$ gamma receptor phagocytosis with the comorbidity between psychiatric symptomatology and substance use in the Mexican population. These results suggest new paradigms for understanding how genetic variability regulates comorbidity.

Received: 28 September 2020; Accepted: 8 March 2021

Published online: 24 March 2021

\section{References}

1. Jané-Llopis, E. \& Matytsina, I. Mental health and alcohol, drugs and tobacco: A review of the comorbidity between mental disorders and the use of alcohol, tobacco and illicit drugs. Drug Alcohol Rev. 25, 515-536 (2006). 
2. Munro, I. \& Edward, K.-L. Mental illness and substance use: An Australian perspective. Int. J. Ment. Health Nurs. 17, 255-260 (2008).

3. Vega, W. A., Sribney, W. M. \& Achara-Abrahams, I. Co-occurring alcohol, drug, and other psychiatric disorders among Mexicanorigin people in the United States. Am. J. Public Health 93, 1057-1064 (2003).

4. Marín-Navarrete, R. et al. Co-occurring disorders: A challenge for Mexican community-based residential care facilities for substance use. J. Dual Diagn. 12, 261-270 (2016).

5. Kessler, R. C. The epidemiology of dual diagnosis. Biol. Psychiatry 56, 730-737 (2004).

6. Medina-Mora, M. E., Borges, G., Benjet, C., Lara, C. \& Berglund, P. Psychiatric disorders in Mexico: Lifetime prevalence in a nationally representative sample. Br. J. Psychiatry 190, 521-528 (2007).

7. Farren, C. K. \& McElroy, S. Treatment response of bipolar and unipolar alcoholics to an inpatient dual diagnosis program. J. Affect. Disord. 106, 265-272 (2008).

8. Gimelfarb, Y. \& Natan, Z. Risk factors for suicide attempts in dual diagnosis patients. Harefuah 148, 355-358, 413 (2009).

9. Oquendo, M. A. et al. Increased risk for suicidal behavior in comorbid bipolar disorder and alcohol use disorders: Results from the National Epidemiologic Survey on Alcohol and Related Conditions (NESARC). J. Clin. Psychiatry 71, 902-909 (2010).

10. Wusthoff, L. E., Waal, H. \& Grawe, R. W. The effectiveness of integrated treatment in patients with substance use disorders cooccurring with anxiety and/or depression-A group randomized trial. BMC Psychiatry 14, 67 (2014).

11. Drake, R. E., Mueser, K. T. \& Brunette, M. F. Management of persons with co-occurring severe mental illness and substance use disorder: Program implications. World Psychiatry 6, 131-136 (2007).

12. Polimanti, R., Agrawal, A. \& Gelernter, J. Schizophrenia and substance use comorbidity: A genome-wide perspective. Genome Med. 9, 25 (2017).

13. Mueser, K. T., Drake, R. E. \& Wallach, M. A. Dual diagnosis: A review of etiological theories. Addict. Behav. 23, 717-734 (1998).

14. Costain, G. \& Bassett, A. S. Clinical applications of schizophrenia genetics: Genetic diagnosis, risk, and counseling in the molecular era. Appl. Clin. Genet. 5, 1-18 (2012).

15. Pasman, J. A. et al. GWAS of lifetime cannabis use reveals new risk loci, genetic overlap with psychiatric traits, and a causal influence of schizophrenia. Nat. Neurosci. 21, 1161-1170 (2018).

16. Duncan, L. et al. Analysis of polygenic risk score usage and performance in diverse human populations. Nat. Commun. 10, 3328 (2019).

17. Williams Amy, A. L. et al. Sequence variants in SLC16A11 are a common risk factor for type 2 diabetes in Mexico. Nature 506, 97-101 (2014).

18. Moreno-Estrada, A. et al. Human genetics. The genetics of Mexico recapitulates Native American substructure and affects biomedical traits. Science 344, 1280-1285 (2014).

19. Wang, S. et al. Geographic patterns of genome admixture in Latin American mestizos. PLoS Genet. 4, e1000037 (2008).

20. Periyasamy, S. et al. Association of schizophrenia risk with disordered niacin metabolism in an Indian genome-wide association study. JAMA Psychiatry 76, 1026-1034 (2019).

21. Martínez-Magaña, J. J. et al. Exploratory analysis of polygenic risk scores for psychiatric disorders: Applied to dual diagnosis. Rev. Invest. Clin. 71, 321-329 (2019).

22. Martínez-Magaña, J. J. et al. The identification of admixture patterns could refine pharmacogenetic counseling: Analysis of a population-based sample in Mexico. Front. Pharmacol. 11, 324 (2020).

23. Reséndiz Escobar, E. et al. National trends in alcohol consumption in Mexico: Results of the National Survey on Drug, Alcohol and Tobacco Consumption 2016-2017. Salud Ment. 41, 7-15 (2018).

24. Pato, M. T. et al. The genomic psychiatry cohort: Partners in discovery. Am. J. Med. Genet. B. Neuropsychiatr. Genet. 162B, 306-312 (2013).

25. Nurnberger, J. I. J. et al. Diagnostic interview for genetic studies. Rationale, unique features, and training. NIMH Genetics Initiative. Arch. Gen. Psychiatry 51, 844-849 (1994).

26. Sheehan, D. V. et al. The Mini-International Neuropsychiatric Interview (M.I.N.I.): The development and validation of a structured diagnostic psychiatric interview for DSM-IV and ICD-10. J. Clin. Psychiatry 59(Suppl 2), 22-57 (1998).

27. Ebbert, J. O., Patten, C. A. \& Schroeder, D. R. The Fagerström test for nicotine dependence-smokeless tobacco (FTND-ST). Addict. Behav. 31, 1716-1721 (2006).

28. Purcell, S. et al. PLINK: A tool set for whole-genome association and population-based linkage analyses. Am. J. Hum. Genet. 81, 559-575 (2007).

29. Marees, A. T. et al. A tutorial on conducting genome-wide association studies: Quality control and statistical analysis. Int. J. Methods Psychiatr. Res. 27, e1608 (2018).

30. Yang, J. et al. Common SNPs explain a large proportion of the heritability for human height. Nat. Genet. 42, 565-569 (2010).

31. Lee, S. H., Wray, N. R., Goddard, M. E. \& Visscher, P. M. Estimating missing heritability for disease from genome-wide association studies. Am. J. Hum. Genet. 88, 294-305 (2011).

32. Yang, J., Lee, S. H., Goddard, M. E. \& Visscher, P. M. GCTA: A tool for genome-wide complex trait analysis. Am. J. Hum. Genet. 88, 76-82 (2011).

33. Price, A. L. et al. Principal components analysis corrects for stratification in genome-wide association studies. Nat. Genet. 38, 904-909 (2006).

34. Conomos, M. P., Miller, M. B. \& Thornton, T. A. Robust inference of population structure for ancestry prediction and correction of stratification in the presence of relatedness. Genet. Epidemiol. 39, 276-293 (2015).

35. Cavalli-Sforza, L. L. The Human Genome Diversity Project: Past, present and future. Nat. Rev. Genet. 6, 333-340 (2005).

36. McLaren, W. et al. The Ensembl variant effect predictor. Genome Biol. 17, 122 (2016).

37. Karczewski, K. J. et al. The mutational constraint spectrum quantified from variation in 141,456 humans. Nature 581, 434-443 (2020).

38. Domingo-Fernández, D., Hoyt, C. T., Bobis-Álvarez, C., Marín-Llaó, J. \& Hofmann-Apitius, M. ComPath: An ecosystem for exploring, analyzing, and curating mappings across pathway databases. npj Syst. Biol. Appl. 5, 1-8 (2019).

39. Goto, S. et al. Organizing and computing metabolic pathway data in terms of binary relations. Pac. Symp. Biocomput. 175-186 (1997).

40. Kanehisa, M., Furumichi, M., Sato, Y., Ishiguro-Watanabe, M. \& Tanabe, M. KEGG: Integrating viruses and cellular organisms. Nucleic Acids Res. 49, D545-D551 (2021).

41. Chen, Y.-A. et al. Integrated pathway clusters with coherent biological themes for target prioritisation. PLoS ONE 9, e99030 (2014).

42. R Development Core Team. R: A language and environment for statistical computing. R Dev. Core Team (2008).

43. Rodríguez-López, M. L. et al. Exploratory analysis of genetic variants influencing molecular traits in cerebral cortex of suicide completers. Am. J. Med. Genet. Part B Neuropsychiatr. Genet. 183, 26-37 (2020).

44. Cabrera, B. et al. Brain gene expression pattern of subjects with completed suicide and comorbid substance use disorder. Mol. Neuropsychiatry 5, 60-73 (2019).

45. Cabrera-Mendoza, B. et al. Brain gene expression-DNA methylation correlation in suicide completers: Preliminary results. Rev. Invest. Clin. https://doi.org/10.24875/RIC.19003250 (2019).

46. Manichaikul, A. et al. Robust relationship inference in genome-wide association studies. Bioinformatics 26, 2867-2873 (2010). 
47. Zhu, Z. et al. Causal associations between risk factors and common diseases inferred from GWAS summary data. Nat. Commun. 9, 224 (2018).

48. Gratten, J. \& Visscher, P. M. Genetic pleiotropy in complex traits and diseases: Implications for genomic medicine. Genome Med. 8, 78 (2016).

49. Anttila, V. et al. Analysis of shared heritability in common disorders of the brain. Science 360, 8757 (2018).

50. Fuller, T. \& Reus, V. Shared genetics of psychiatric disorders [version 1; peer review: 2 approved]. F1000Research 8 (2019).

51. Davis, L. K. Common knowledge: Shared genetics in psychiatry. Nat. Neurosci. 22, 331-332 (2019).

52. Corponi, F. et al. Genetic basis of psychopathological dimensions shared between schizophrenia and bipolar disorder. Prog. NeuroPsychopharmacol. Biol. Psychiatry 89, 23-29 (2019).

53. Schwabe, I. et al. Unraveling the genetic architecture of major depressive disorder: Merits and pitfalls of the approaches used in genome-wide association studies. Psychol. Med. 49, 2646-2656 (2019).

54. Cai, N. et al. Minimal phenotyping yields GWAS hits of reduced specificity for major depression. bioRxiv https://doi.org/10.1101/ 440735 (2018).

55. Jones, S. E. et al. Genome-wide association analyses of chronotype in 697,828 individuals provides insights into circadian rhythms. Nat. Commun. 10, 343 (2019).

56. Jansen, P. R. et al. Genome-wide analysis of insomnia in 1,331,010 individuals identifies new risk loci and functional pathways. Nat. Genet. 51, 394-403 (2019).

57. Watanabe, K. et al. A global overview of pleiotropy and genetic architecture in complex traits. Nat. Genet. 51, 1339-1348 (2019).

58. Krystal, J. H. \& State, M. W. Psychiatric disorders: Diagnosis to therapy. Cell 157, 201-214 (2014).

59. D'Souza, M. S. Glutamatergic transmission in drug reward: Implications for drug addiction. Front. Neurosci. 9, 404 (2015).

60. Pivovarova, N. B. \& Andrews, S. B. Calcium-dependent mitochondrial function and dysfunction in neurons: Minireview. FEBS J. 277, 3622-3636 (2010).

61. Nicholls, D. G. \& Budd, S. L. Mitochondria and neuronal glutamate excitotoxicity. Biochim. Biophys. Acta Bioenerg. 1366, 97-112 (1998).

62. Choi, D. W. Glutamate neurotoxicity in cortical cell culture is calcium dependent. Neurosci. Lett. 58, 293-297 (1985).

63. Abele, A. E., Scholz, K. P., Scholz, W. K. \& Miller, R. J. Excitotoxicity induced by enhanced excitatory neurotransmission in cultured hippocampal pyramidal neurons. Neuron 4, 413-419 (1990).

64. Coyle, J. T. \& Puttfarcken, P. Oxidative stress, glutamate, and neurodegenerative disorders. Science 262, 689-695 (1993).

65. Gass, J. T. \& Olive, M. F. Glutamatergic substrates of drug addiction and alcoholism. Biochem. Pharmacol. 75, 218-265 (2008).

66. Teng, S. et al. Rare disruptive variants in the DISC1 Interactome and Regulome: Association with cognitive ability and schizophrenia. Mol. Psychiatry 23, 1270-1277 (2018).

67. Camargo, L. M., Wang, Q. \& Brandon, N. J. What can we learn from the disrupted in schizophrenia 1 interactome: LESSONS for target identification and disease biology? in Growth Factors and Psychiatric Disorders, Vol. 289, 208-216 (Wiley, 2008).

68. Camargo, L. M. et al. Disrupted in Schizophrenia 1 interactome: Evidence for the close connectivity of risk genes and a potential synaptic basis for schizophrenia. Mol. Psychiatry 12, 74-86 (2007).

69. Trossbach, S. V. et al. Peripheral DISC1 protein levels as a trait marker for schizophrenia and modulating effects of nicotine. Behav. Brain Res. 275, 176-182 (2014).

70. Daws, L. C. et al. Insulin signaling and addiction. Neuropharmacology 61, 1123-1128 (2011).

71. Owens, W. A. et al. Deficits in dopamine clearance and locomotion in hypoinsulinemic rats unmask novel modulation of dopamine transporters by amphetamine. J. Neurochem. 94, 1402-1410 (2005).

72. Robertson, S. D. et al. Insulin reveals Akt signaling as a novel regulator of norepinephrine transporter trafficking and norepinephrine homeostasis. J. Neurosci. 30, 11305-11316 (2010).

73. Huang, X. F., Yu, Y., Zavitsanou, K., Han, M. \& Storlien, L. Differential expression of dopamine D2 and D4 receptor and tyrosine hydroxylase mRNA in mice prone, or resistant, to chronic high-fat diet-induced obesity. Mol. Brain Res. 135, 150-161 (2005).

74. Li, C.-Y., Mao, X. \& Wei, L. Genes and (common) pathways underlying drug addiction. PLoS Comput. Biol. 4, e2 (2008).

75. Kleinridders, A. \& Pothos, E. N. Impact of brain insulin signaling on dopamine function, food intake, reward, and emotional behavior. Curr. Nutr. Rep. 8, 83-91 (2019).

76. Volkow, N. D. et al. "Nonhedonic" food motivation in humans involves dopamine in the dorsal striatum and methylphenidate amplifies this effect. Synapse 44, 175-180 (2002).

77. Liu, S. et al. Consumption of palatable food primes food approach behavior by rapidly increasing synaptic density in the VTA. Proc. Natl. Acad. Sci. U.S.A. 113, 2520-2525 (2016).

78. Liu, S., Labouèbe, G., Karunakaran, S., Clee, S. M. \& Borgland, S. L. Effect of insulin on excitatory synaptic transmission onto dopamine neurons of the ventral tegmental area in a mouse model of hyperinsulinemia. Nutr. Diabetes 3, e97-e97 (2013).

79. Mebel, D. M., Wong, J. C. Y., Dong, Y. J. \& Borgland, S. L. Insulin in the ventral tegmental area reduces hedonic feeding and suppresses dopamine concentration via increased reuptake. Eur. J. Neurosci. 36, 2336-2346 (2012).

80. Zhao, Z. et al. Transcriptome sequencing and genome-wide association analyses reveal lysosomal function and actin cytoskeleton remodeling in schizophrenia and bipolar disorder. Mol. Psychiatry 20, 563-572 (2015).

81. Staretz-Chacham, O., Choi, J. H., Wakabayashi, K., Lopez, G. \& Sidransky, E. Psychiatric and behavioral manifestations of lysosomal storage disorders. Am. J. Med. Genet. B Neuropsychiatr. Genet. 153, 1253-1265 (2010).

\section{Acknowledgements}

We wish to thank the work committee and the field coordination team of the Encodat 2016, headed by Aurora Franco. We are also grateful to the microarray unit of the Instituto Nacional de Medicina Genómica, and Rául Mojica Espinoza and the expression and microarray unit of INMEGEN, for technical assistance with microarray processing. José Jaime Martínez-Magaña is a doctoral student from theBiomedical Sciences Program at Juárez Autonomous University of Tabasco and was supported by CONACyT (No. 703003).

\section{Author contributions}

J.J.M.M., A.D.G.M., and I.E.J.R. performed the analysis and wrote the first version of the manuscript. J.J.M.M. and J.A.V.V. performed the bioinformatics and statistical analyses. J.J.M.M., A.D.G.M., B.C.M., and A.A. carried out the DNA extraction and microarray preparation. M.B.G., I.E.J.R., C.A.T.Z., E.S., E.Sa., O.R.M., C.F.B., and T.B.G.C. carried out data acquisition and clinical evaluation. H.N. and M.E.M.M. conceptualized and designed the study. All authors reviewed the manuscript.

\section{Funding}

This study received funding from the Instituto Nacional de Medicina Genómica (Grant Nos. 23/2015/I and 06/2018/I), and from the Consejo Nacional de Ciencia y Tecnología (CONACyT) 2016 Fund for the Development 
of Scientific Projects to Address National Problems (Grant No. PN22296). The fieldwork for the surveys was funded in 2016 by the Comisión Nacional contra las Adicciones (CONADIC).

\section{Competing interests}

The authors declare no competing interests.

\section{Additional information}

Supplementary Information The online version contains supplementary material available at https://doi.org/ 10.1038/s41598-021-85881-4.

Correspondence and requests for materials should be addressed to H.N.

Reprints and permissions information is available at www.nature.com/reprints.

Publisher's note Springer Nature remains neutral with regard to jurisdictional claims in published maps and institutional affiliations.

(c) (i) Open Access This article is licensed under a Creative Commons Attribution 4.0 International License, which permits use, sharing, adaptation, distribution and reproduction in any medium or format, as long as you give appropriate credit to the original author(s) and the source, provide a link to the Creative Commons licence, and indicate if changes were made. The images or other third party material in this article are included in the article's Creative Commons licence, unless indicated otherwise in a credit line to the material. If material is not included in the article's Creative Commons licence and your intended use is not permitted by statutory regulation or exceeds the permitted use, you will need to obtain permission directly from the copyright holder. To view a copy of this licence, visit http://creativecommons.org/licenses/by/4.0/.

(C) The Author(s) 2021 